\title{
Spectral statistics in disordered metals: a trajectories approach
}

\author{
R. A. Smith ${ }^{1}$, I. V. Lerner ${ }^{1}$, and B. L. Altshuler ${ }^{2,3}$ \\ ${ }^{1}$ School of Physics and Space Research, University of Birmingham, Edgbaston, Birmingham B15 2TT, United Kingdom \\ ${ }^{2}$ NEC Research Institute, 4 Independence Way, Princeton, NJ 08540, USA \\ ${ }^{3}$ Physics Department, Princeton University, Princeton, NJ 08544, USA
}

(January 22, 1998)

\begin{abstract}
We show that the perturbative expansion of the two-level correlation function, $R(\omega)$, in disordered conductors can be understood semiclassically in terms of self-intersecting particle trajectories. This requires the extension of the standard diagonal approximation to include pairs of paths which are non-identical but have almost identical action. The number of diagrams thus produced is much smaller than in a standard field-theoretical approach. We show that such a simplification occurs because $R(\omega)$ has a natural representation as the second derivative of free energy $F(\omega)$. We calculate $R(\omega)$ to 3-loop order, and verify a one-parameter scaling hypothesis for it in $2 \mathrm{~d}$. We discuss the possibility of applying our "weak diagonal approximation" to generic chaotic systems.
\end{abstract}

PACS numbers: 73.23.-b, 73.20.Fz, 05.45+b

The relationship between the quantum properties of disordered and classically chaptic systems has been the focus of much recent research 6 . The main reason for this is that both types of system show the same underlying behavior - their energy spectra in appropriate regions have statistics given by random matrix theory (RMT) 6]. The spectral properties in these universal regions are well understood. The challenge now is to understand whether generic features also emerge in the deviations from universality. Answering this question is made difficult because two very different languages are used to characterize the two types of system. In quantum disordered systems one averages over all realizations of disorder to obtain an effective field theory: the nonlinear sigma model 10 . In quantum chaotic systems, (i.e. quantum systems which are chaotic in the classical limit), one considers particular system using the Gutzwiller trace formula11, which involves a sum over classical periodic orbits. Each language has its own strengths and weaknesses. The field theory technique is rather formal and somewhat opaque physically, but has a well-defined perturbation expansion. The trace formula appears to be more physically transparent in that one is summing over classical trajectories. However, this sum is difficult to perform, its convergence properties are not well understood, and no controlled expansion is currently available. It is therefore natural that one should attempt to use the strengths of one language to compensate the weaknesses of the other.

One recent attempt was to try to carry over the powerful calculational techniques dereloped for the disordered systems to the chaotic systems 1.5 . In the most consistent form, one averages over a certain energy interval to generate a field-theoretical functional in the Wigner representation (the "ballistic" sigma-model). From this one can derive, e.g., the two-level correlation function for quantum chaotic systems. In the universal ergodic regime, the non-perturbative derivation is equivalent to one which Efetov developed for disordered systems, as expected.
In addition the leading-order perturbative term目 in the non-ergodic regime gives a result equivalent to that obtained in the diagonal approximation 12 to the Gutzwiller trace formula11. The same approximation when applied to the disordered systems 1 also gives the leading order perturbative term first derived in Ref. 13. One therefore expects that higher order perturbative terms in quantum chaotic systems would be analogous to those occurring in disordered systems (where they are known as weaklocalization corrections). The ballistic sigma model is, however, ill-defined in the ultraviolet limit, making perturbative analysis currently ambiguous.

On the other hand, various authors14 16 . ceeded in the opposite direction, and developed semiclassical methods for the quantum disordered system. The goal is to gain a more intuitive picture of phenomena such as weak localization and universal conductance fluctuations.

In this paper we show how the diagrammatics for spectral correlations in disordered conductors can be rewritten in terms of particle trajectories which self-intersect in real space. Each diagram for the two-level correlation function is represented as consisting of two trajectories that are identical everywhere except at selfintersection regions, where they are rejoined in different ways. As the two trajectories are identical for most of their length, they are phase coherent, and interfere constructively. The perturbation parameter, $1 / g$, where $g$ is the the dimensionless conductance, can be understood as the probability for a self-intersection to occur. The great advantage of this approach is the drastic reduction in the number of diagrams which occur in a given order of perturbation theory This reduction arises because our approach does not distinguish the starting points of the (closed) trajectories, whereas the standard approach does. We show that when translated into field theory language, this means that the two-level correlation function, $R(\omega)$, is the second derivative with respect to energy, $\omega$, of the free energy, $F(\omega)$. As a particular problem we 
calculate the two-level correlation function to three-loop order in perturbation theory, which yields the leading order level statistics for the unitary system in 2 d. Finally we show that the three-loop results for $R(\omega)$ in both unitary and orthogonal systems can be derived from a oneparameter scaling hypothesis in which the renormalized conductance $g(\omega)$ is substituted into the one-loop result. Note that in our approach we are using the trajectory picture to classify the field theory diagrammatics in contrast to the work discussed in the previous paragraph where field theory is being used to classify periodic orbits.

We consider the relation of the picture of trajectories to the perturbation expansion of $R(\omega)$ which is defined by

$$
R(\omega)=\frac{1}{\nu^{2}}\langle\nu(E+\omega) \nu(E)\rangle-1 .
$$

Here $\nu(E)$ is the density of states per unit volume which can be written, given the spectrum $\left\{E_{n}\right\}$ of the system, as

$$
\nu(E)=\frac{1}{L^{d}} \sum_{n} \delta\left(E-E_{n}\right),
$$

and $\nu=\langle\nu(E)\rangle=1 /\left(\Delta L^{d}\right)$, where $\Delta$ is the mean level spacing. Here $\langle\ldots\rangle$ denotes the averaging, either over all realizations of disorder for a disordered system or over a certain energy window for a chaotic system. Such energy windows are always narrow enough so that $\nu$ has no energy dependence. The semiclassical approach allows one to write $\nu(E)$ as a sum over classical paths by means of the Gutzwiller trace formula11,

$$
\nu(E)=\sum_{p} A_{p}(E) \exp \left(i S_{p}(E) / \hbar\right)
$$

where $A_{p}(E)$ and $S_{p}(E)$ are the amplitude and action of the $p$-th periodic orbit at energy $E$. One can then substitute this semiclassical formula into the definition of the two-level correlation function, $R(\omega)$, and take the Fourier transform to obtain the spectral form factor, $K(t)$,

$$
K(t)=\int \frac{d \omega}{2 \pi \hbar} \exp (-i \omega t / \hbar) R(\omega) .
$$

Since all factors vary slowly with $E$ except the action $S_{p}(E)$, one expands $S_{p}(E+\omega)$ to first order to obtain

$$
K(t)=\sum_{p, q} A_{p} A_{q}^{*} e^{i\left(S_{p}-S_{q}\right) / \hbar} \delta\left[t-\frac{1}{2}\left(T_{p}+T_{q}\right)\right]
$$

where $T_{p}(E)=d S_{p}(E) / d E$ is the period of the orbit $p$.

To proceed further Berry 12 introduced the diagonal approximation assuming that only terms with $p=q$ contribute to the sum in Eq. (5). The reasoning is that terms with $p \neq q$ have randomly varying phases and cancel each other. In the disprdered metal such a diagonal approximation was shown to-reproduce the leading order perturbative contribution 13 to $R(\omega)$.
The question that we address here is how to get regular corrections to the diagonal approximation which are due to contributions of relatively long trajectories. Such corrections are of fundamental importance, especially for two-dimensional disordered systems: the leading order term ferresponding to the diagonal approximation vanishes 17 for $d=2$. However, the possibility of getting the regular corrections could be interesting in a much wider context. To evaluate the corrections one has to consider pairs of paths with almost equal actions, $S_{p} \approx S_{q}$, like in the diagonal approximation, but allow $p \neq q$.

To construct the weak diagonal approximation which allows for such pairs of trajectories we start with a path that self-intersects in real space at one or more points. At a point of intersection we break up the path and make different trajectories by joining the pieces together in different ways. We see at once that this gives rise to a perturbation expansion, with the perturbative order given by the number of loops in real space created by selfintersections, and the perturbation parameter given by the probability of having a self-intersection. In the case of a disordered electronic system, we will show that this expansion is none other than the usual field-theoretical loop expansion. Before we do this, let us speculate on the nature of this expansion for a generic chaotic system.

In this case the above picture of self-intersecting trajectories requires clarification. First of all, the picture of trajectories is formulated in phase space rather than in real space. Of course, classical trajectories which are identical along part of their length in phase space cannot diverge in the way discussed above - this process is quantum mechanical in nature. The uncertainty principle means that phase space is coarse-grained into boxes with size of order $\hbar^{d}$. Two trajectories which were originally nearly identical (i.e. passing through the same phase space boxes) start to deviate significantly at the Ehrenfest time, $t_{\mathrm{Ehr}}$, due to quantum processes such as diffraction. Then we can make pairs of piecewise identical trajectories as described above. For this picture to make sense we need to work at time-scales greater than $t_{\mathrm{Ehr}}$. This has been noted in Ref. 18 which recently considered the weak localization correction in a disordered system where the Ehrenfest time is determined by the diffraction at randomly distributed scattering spheres. In the usual model of a disordered conductor with point-like scatterers $t_{\mathrm{Ehr}}$ coincides with the elastic scattering time $\tau$ since the particle's direction is totally randomized after each scattering. In general, we expect that the classification of trajectories in the weak diagonal approximation might be valid for chaotic system with $t_{\mathrm{Ehr}} \ll t_{\mathrm{erg}}$ where $t_{\mathrm{erg}}$ is the ergodic time scale at which the trajectory samples the entire phase space. This condition is necessary in order to treat the self-intersection region as a perturbation. It simply means that the length of almost identical regions of the two trajectories is much larger than the length of the self-intersection region. 
At time scales $t \gg t_{\mathrm{erg}}$, one expects completely universal havior which is described by the random matrix theory. 19 This has been conjectured by Bohigas et alt and partially proved by Andreev et al 0.6 This universal behavior is completely non-perturbative and cannot be described by the diagonal approximation. Moreover, even for $t \ll t_{H}$, where $t_{H} \equiv \hbar / \Delta$ is the Heisenberg time, the diagonal approximation does not reproduce the deviation from the linear behavior of $K(t)$ known from the random matrix theory. We will show that we can exactly allow for this deviation within our weak diagonal approximation.

At time scales $t_{\mathrm{Ehr}} \ll t \ll t_{\text {erg }}$, one expects nonuniversal corrections to the diagonal approximation. It is not clear whether this is true for a generic chaotic system. Attempts2 to generate these corrections within the ballistic nonlinear $\sigma$ model 6 have led to results vanishing to all orders in perturbation theory. These results are dubious, however, because they depend strongly on the short-time regularization procedure. In particular, there exist regularizations where the corrections do not vanish and are totally governed by short-time processes at time scales $t \lesssim t_{\mathrm{Ehr}}$. In addition, the ballistic $\sigma$ model itself might not take into account all relevant quantum processes.

In the disordered conductor these corrections can be calculated both for $t \gg t_{\mathrm{erg}}$ and for $t \ll t_{\mathrm{erg}}$. Here $t_{\text {erg }}=L^{2} / D$ is the time taken for an electron to diffuse to the boundaries. We will show that the trajectory picture is naturally related to a standard diagrammatic expansion and, moreover, allows considerable simplifications in this expansion.

We will now draw all closed trajectories with up to two self-intersections regions and show their relation to the diagrams up to three-loop order. Let us first rewrite a pair of identical (up to discrete symmetries of the ensemble) trajectories which correspond to Berry's diagonal approximation, 12 as a diagram in field theory language.

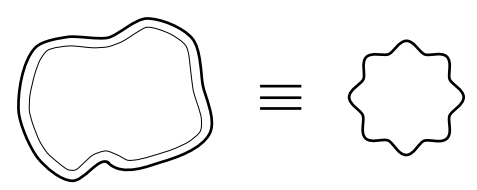

(a)

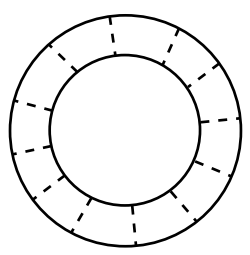

(b)
FIG. 1. (a) The 1-loop diagram for the free energy $F(\omega)$, which consists of pairs of trajectories that are identical up to time reversal symmetry. In the field theory language it consists of a single closed wavy line. (b) The same diagram in the disordered metal. Since the closed wavy line in (a) consists of a sum over impurity ladders, and the diagram with $n$ impurity ladders has symmetry factor $1 / n$, the ladder gives logarithmic contribution $-\ln \left(D q^{2}-i \omega\right)$.

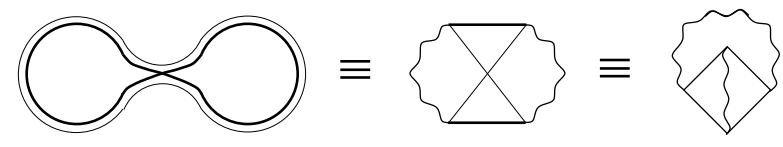

FIG. 2. The 2-loop diagram for the free energy $F(\omega)$ corresponding to paths with one self-intersection. First we show the two different ways of linking up the paths at the selfintersection point. Then we rewrite this in the field theory language so that regions where the two paths are identical become wavy lines, whilst the region of self-intersection becomes a closed box. Finally we twist the latter diagram into its usual form.

The pair of identical or time-reversed trajectories becomes a diffusion or Cooperon propagator, respectively, drawn as a wavy line. This yields the one-loop diagram shown in Fig. 1a.

The two-loop case is shown in Fig. 2 and involves one self-intersection region. At this point the paths can be linked up in two different ways, and the diagram consists of these two trajectories. Again we rewrite in field theory language with the identical or time-reversed portions of path becoming diffusion or Cooperon propagators. The region around the self-intersection becomes an effective interastion between these propagators known as a Hikami box 21 . If we put arrows on the trajectories to show their direction of traversal we see that they go in the same direction over part of the diagram. This means that time-reversal invariance is required for their actions to be identical. Hence there is no two-loop term in the unitary case, where time-reversal invariance is broken; in fact, all even loop contributions vanish for the same reason.

The three-loop case is shown in Fig. 3, and involves two self-intersections. The situation here is more complicated since there are three distinct topological ways for two self-intersections to occur. They can occur either at the same point, or at two different points $A$ and $B$. In the latter case there are two distinct orderings in which the trajectory can be traversed $-A A B B$ and $A B A B$. Finally there is more than one way of linking up the partial paths to form trajectories. There are five diagrams in total: $F_{3 a}$ has $A A B B$ form; $F_{3 b}$ and $F_{3 c}$ have intersection at only one point; $F_{3 d}$ and $F_{3 e}$ have $A B A B$ form. Putting arrows on the trajectories we find that only $F_{3 b}$ and $F_{3 d}$ contribute to the unitary case.

We see that the above procedure can obviously be generalized to any order of perturbation theory, and is a powerful way of ensuring that all contributions have been considered.

Let us now relate these pictures of semiclassicat trajectories to the standard diagrammatic approach 13 for evaluating $R(\omega)$. The starting point is the expression for the density of states, Eq. (2), in terms of electron Green's functions, 

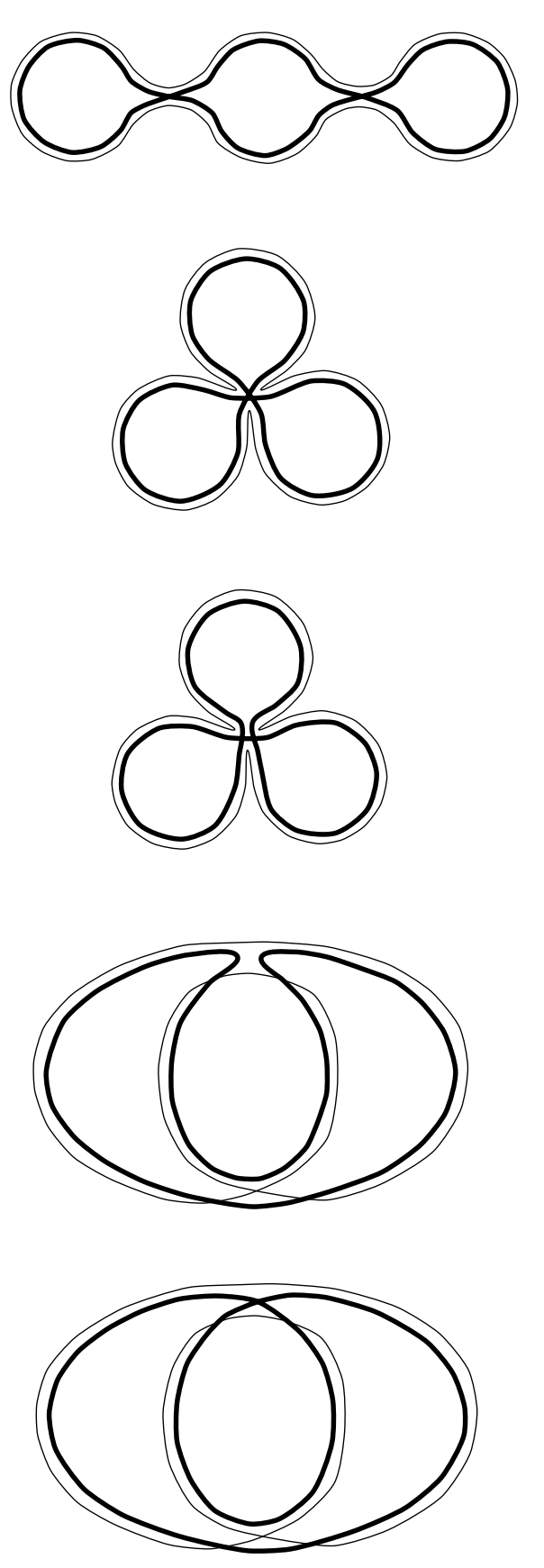
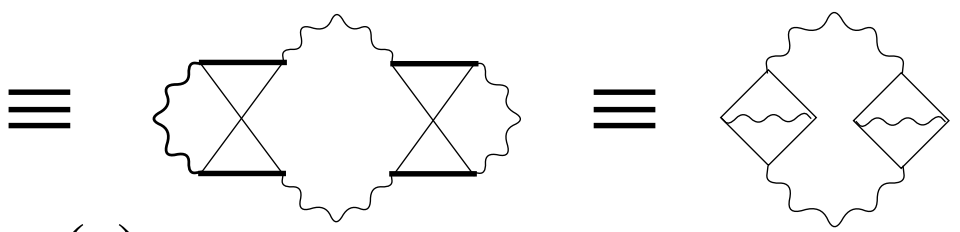

(a)

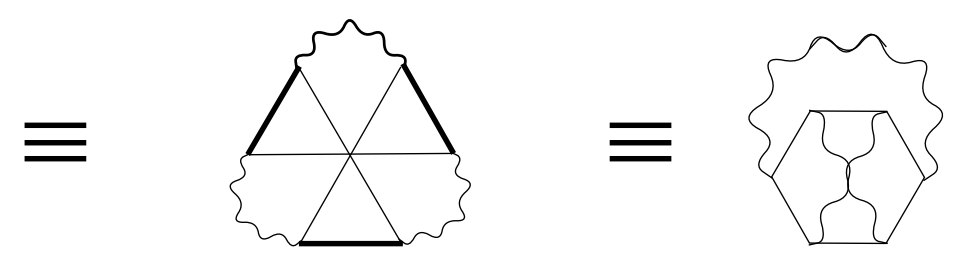

(b)
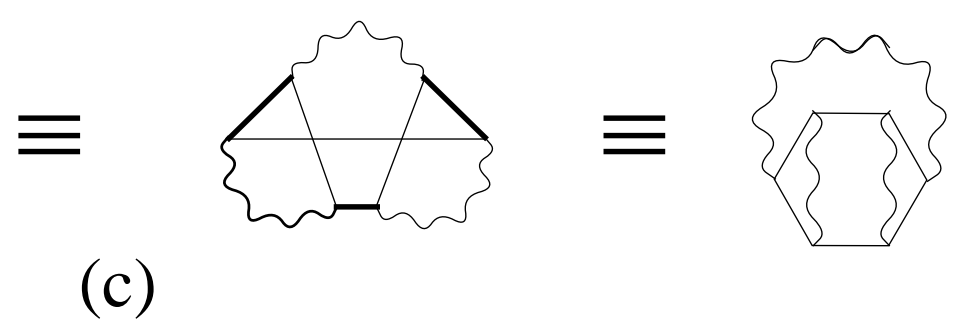

(c)
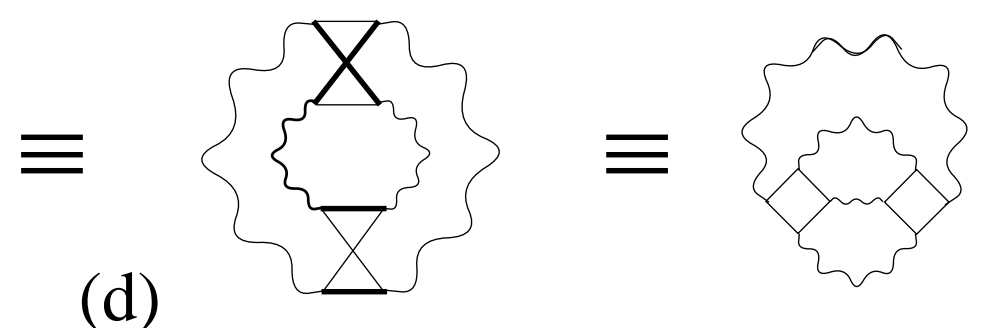

$\overline{\bar{\equiv}}$
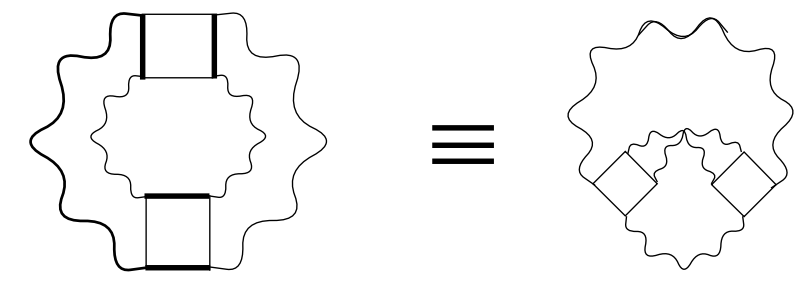

FIG. 3. The five 3-loop diagrams for the free energy $F(\omega)$ corresponding to paths with two self-intersections. We follow the format of Fig. 2, showing first the pairs of distinct trajectories; then rewriting these with wavy lines representing regions where the two paths are identical, and closed boxes at the self-intersection points; then finally twisting the latter diagrams in their usual form. 


$$
\nu(E)=\frac{i}{2 \pi L^{d}} \int \mathrm{d}^{d} r\left[G^{R}(\boldsymbol{r}, \boldsymbol{r} ; E)-G^{A}(\boldsymbol{r}, \boldsymbol{r} ; E)\right] .
$$

It follows from this expression and the definition (1) that

$$
R(\omega)=\frac{\Delta^{2}}{2 \pi^{2}} \Re \mathrm{e} \int \mathrm{d}^{d} r \mathrm{~d}^{d} r^{\prime}\left\langle G^{R}\left(\boldsymbol{r}, \boldsymbol{r} ; E_{1}\right) G^{A}\left(r^{\prime}, r^{\prime} ; E_{2}\right)\right\rangle_{\mathrm{c}}, \quad \omega \equiv E_{1}-E_{2} .
$$

Here only the connected average of $G^{R} G^{A}$ remains: the sum of all unconnected averages is absorbed by -1 in the definition (1), while the connected average of $G^{R} G^{R}+G^{A} G^{A}$ vanishes.

Equation (7) directly corresponds to the semiclassical expressions (4) and (5), since $G^{R}(\boldsymbol{r}, \boldsymbol{r} ; E)$ is the quantum mechanical propagator for a particle of energy $E$ to start and finish at the same point $r$. We can then represent $G^{R}(\boldsymbol{r}, \boldsymbol{r} ; E)$ as the sum of all closed paths while $G^{A}(\boldsymbol{r}, \boldsymbol{r} ; E)$ will be the sum of all closed paths traversed in the opposite direction. Averaging over disorder results in the vanishing of all contributions apart from those corresponding to pairs of coherent paths. This coherence arises when the pair of paths involves scattering off the same impurities although not necessarily in the same order. Pictorially, scattering off the same impurities is shown by impurity lines between $G^{R}$ and $G^{A}$. In the lowest perturbative order, the closed paths for $G^{R}$ and $G^{A}$ are identical, apart from their different starting points $\mathbf{r}$ and $\mathbf{r}^{\prime}$, while the impurity lines form a ladder which corresponds to a diffusion propagator

$$
P(q, \omega)=\frac{1}{2 \pi \nu \tau^{2}} \frac{1}{D q^{2}-i \omega}, \quad q \ell \ll 1, \omega \tau \ll 1
$$

or its time-reversed counterpart, a Cooperon.

The key feature of the diagram for $R(\omega)$ generated in such a way, Fig. 4 , is that the starting points of the two

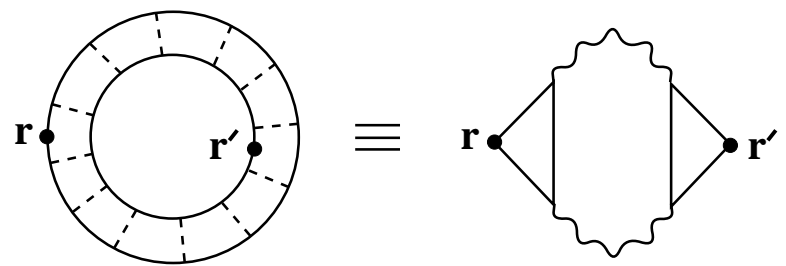

FIG. 4. The field theory diagram for the lowest order contribution to the two-level correlation function $R(\omega)$ in the standard approach. This is identical to Fig. 1 except that the starting points of the two trajectories, $\mathbf{r}$ and $\mathbf{r}^{\prime}$ are distinguished. In general inserting points in such a way yields many more diagrams for $R(\omega)$ than for $F(\omega)$. paths, $\mathbf{r}$ and $\mathbf{r}^{\prime}$, are fixed in the beginning, even though we finally integrate over all $\mathbf{r}$ and $\mathbf{r}^{\prime}$ in Eq. (7). Contrarily, there are no starting points on the semiclassical diagrams of Fig. 1. Obviously, one can obtain the diagram of Fig. 4 from the semiclassical picture of Fig. 1 by inserting points $\mathbf{r}$ and $\mathbf{r}^{\prime}$ into different electron lines in all possible ways. Inserting an external point is equivalent to taking a derivative with respect to energy: inserting $\mathbf{r}$ replaces $G^{R}\left(E_{1}\right)$ by $G^{R}\left(E_{1}\right) G^{R}\left(E_{1}\right)$, which can be achieved by the action of $-\partial / \partial E_{1}$. Similarly, inserting $\mathbf{r}^{\prime}$ gives the same result as $-\partial / \partial E_{2}$. After averaging $R(\omega)$ depends only upon $\omega=E_{1}-E_{2}$. Therefore, one may represent it as the second derivative of a certain function $F(\omega)$,

$$
R(\omega)=-\Delta^{2} \frac{\partial^{2}}{\partial \omega^{2}} F(\omega)
$$

We will show below that this $F(\omega)$ corresponds to the 'free energy' of the appropriate field-theoretical functional. Note that such a representation of $R(\omega)$ has previously been used in the gvaluation of first-order diagrams in the ballistic regime22. Here we show that Eq. (9) is valid to all orders of perturbation theory.

Higher order perturbative contributions to $R(\omega)$ arise from two paths where scattering occurs from the same impurities but in a different order. The parts of the diagrams where the sequence of impurity scatterings coincides (or is time reversed) for the two paths are again represented by diffuson (or Cooperon) ladders. These ladders are connected by Hikami boxes which represent the change in the scattering sequence. In terms of paths, this corresponds to the regions of self-intersection described above. The only difference between these higher order contributions to $R(\omega)$ and semiclassical diagrams in Figs. 2 and 3 is the necessity to distinguish starting points $\mathbf{r}$ and $\mathbf{r}^{\prime}$. Therefore these semiclassical diagrams describe higher order contributions to $F(\omega)$, and $R(\omega)$ can be obtained with the help of Eq. (9).

Finally let us further justify the above pictorial discussion with a rigorojs derivation using the standard fieldtheory machinery 0.2123 . We perform the average over disorder by the replica trick. This can be done with either bosonic (commuting) or fermionic (anticommuting) variables, and both must yield the same results for physical quantities. In this paper we will use bosonic variables. The two-level correlation function is then given by

$$
R(\omega)=-\lim _{N \rightarrow 0} \frac{\Delta^{2}}{16 \pi^{2} N^{2}} \int \mathcal{D} Q\left(\int \mathrm{d}^{d} r \operatorname{Tr}[\Lambda Q(\boldsymbol{r})]\right)^{2} \exp (-\mathcal{F}[Q ; \omega])-1
$$


where $\mathcal{F}[Q ; \omega]$ is the non-linear sigma model functional

$$
\mathcal{F}[Q ; \omega]=\frac{\pi \nu}{8} \int \mathrm{d}^{d} r \operatorname{Tr}\left[D(\nabla Q)^{2}-2 i \omega \Lambda Q\right]
$$

The matrix $Q$ has rank $2 N$ : we made $N$ replicas each for retarded and advanced Green's functions. It satisfies the standard saddle-point conditions:

$$
Q^{2}=I, \quad \operatorname{Tr}(Q)=0 .
$$

The matrix $\Lambda$ is diagonal with elements +1 for retarded indices and -1 for advanced indices. Then it follows that

$$
\operatorname{Tr}(\Lambda Q(\boldsymbol{r}))=N\left(G^{R}-G^{A}\right)=-2 \pi i N \nu(r),
$$

so that the prefactor in Eq. (10) is the product of two densities of states. If we now introduce the free energy $F(\omega)$ as

$$
F(\omega)=\lim _{N \rightarrow 0} \frac{1}{N^{2}} \int \mathcal{D} Q \exp (-\mathcal{F}[Q ; \omega]),
$$

we see from Eqs. (10) and (11) that $R(\omega)$ can indeed be written in terms of $F(\omega)$ as in Eq. (9).

We can now apply the usual perturbative methods to expand $F(\omega)$ in powers of the coupling constant (inverse dimensionless conductance) $1 / g \equiv 1 / 4 \pi^{2} \nu D$ and then apply the relation (9) to obtain $R(\omega)$. The great advantage of this method is the much smaller number of diagrams we need to evaluate. For example, in three-loop perturbation theory for the orthogonal case there are 5 diagrams for $F(\omega)$ compared to 41 for $R(\omega)$.

In previous work 23 a source field for $\nu(E)$ has been introduced with a complex index structure similar to that necessary for calculating the conductance moments. A considerable simplification here is that $\omega$ itself suffices as a source field for calculating $\nu(E)$. We note that this method cannot be extended to the supersymmetric sigma model 3 where the free energy is always unity owing to the fact that the supersymmetry is preserved in the effective functional. To break the supersymmetry one must introduce the $k$-matrix in the expression for the prefactors, $\operatorname{STr}(k \Lambda Q)$, or equivalently introdwce an appropriate source term in the effective functionale. Although this is straightforward, all 'savings' in terms of the number of diagrams would be lost. Thus, for perturbative calculations the replicated $\sigma$ model turns out to be more economical than the supersymmetric one. Finally we note that the free energy has previously been calculated up to four-loop order 24 in order to find $\beta(g)$. Our result above gives a direct physical meaning to the field-theoretic free energy.
To generate perturbation theory we must introduce a parameterization of $Q$ that satisfies the saddle-point constraints. There are several parameterizations available, and they will give different contributions for a given diagram, but the sum of all contributions at a given order of perturbation theory will always be the same. The particular one used depends upon the application. The choice

$$
Q=\Lambda\left(W+\sqrt{1+W^{2}}\right) \quad W=\left(\begin{array}{cc}
0 & 2 i V \\
2 i V^{+} & 0
\end{array}\right)
$$

reproduces the results of the griginal impurity diagrammatics in the diffusive regime 21 , and is thus useful for direct comparison between the results of impurity diagrammatics and the non-linear $\sigma$-model. Here $V$ is an unconstrained $N \times N$ matrix with elements of the form appropriate for the given Dyson ensemble. The functional integration is then carried out over the independent variables $V$. Another parameterization, 23

$$
Q=\Lambda(1+W / 2)(1-W / 2)^{-1}
$$

where $W$ has the same properties as before, is more convenient for calculation because many terms then vanish, and the Jacobian of the transition from $Q$ to $W$ is unity in the replica limit. Since the Jacobian contributes terms necessary to remove ultraviolet divergences, this means that the sum of all terms at any order in perturbation theory will have no ultraviolet divergence and so there is no need for regularization. Considerations in the ergodic regime are also much simpler, since Jacobian terms would contribute there. In what follows we will, therefore, use these parameterization.

When we substitute a parameterization into the free energy functional $\mathcal{F}[Q]$ we obtain a sum of vertices,

$$
\mathcal{F}[Q]=\sum_{n=1}^{\infty} \mathcal{F}_{2 n}[W]
$$

where the $\mathcal{F}_{2 n}[W]$ vertex contains $2 n$ powers of $W$. The first term $\mathcal{F}_{2}$ is the quadratic part, and leads to the ladder propagators. The perturbation expansion up to the third loop order is then obtained by expanding out the exponential in the other $\mathcal{F}_{2 n}$,

$$
\exp \left(-\mathcal{F}_{4}-\mathcal{F}_{6}-\mathcal{F}_{8}-\ldots\right)=1-\mathcal{F}_{4}-\left(\mathcal{F}_{6}-\mathcal{F}_{4}^{2} / 2\right)
$$

and averaging with respect to $\exp \left(-\mathcal{F}_{2}\right)$. Upon substituting the parameterization we get

$$
\begin{aligned}
& \mathcal{F}_{2}=\pi \nu \int d^{d} r \operatorname{Tr}\left[-D \nabla V \nabla V^{+}-i \omega V V^{+}\right] \\
& \mathcal{F}_{4}=\pi \nu \int d^{d} r \operatorname{Tr}\left[-2 D \nabla V \nabla V^{+} V V^{+}-i \omega V V^{+} V V^{+}\right] \\
& \mathcal{F}_{6}=\pi \nu \int d^{d} r \operatorname{Tr}\left[D\left(2 \nabla V \nabla V^{+} V V^{+}+\nabla V V^{+} V \nabla V^{+} V V^{+}\right)-i \omega V V^{+} V V^{+} V V^{+}\right]
\end{aligned}
$$


If we then Fourier transform into momentum space we recover the expected diffusion propagator of Eq. (8) as the average of $V V^{+}$with respect to $\exp \left(-\mathcal{F}_{2}\right)$,

$$
\left\langle V_{i \alpha}(q) V_{\beta j}^{+}(-q)\right\rangle=\frac{1}{2 \pi \nu} \frac{1}{D q^{2}-i \omega} \delta_{i j} \delta_{\alpha \beta}
$$

The higher-order terms yield the effective four- and sixpoint vertices

$$
\begin{aligned}
& V_{4}\left(q_{i}\right)=2 \pi \nu\left[-D \Delta_{4}^{1}-2 i \omega\right] \\
& V_{6}\left(q_{i}\right)=-2 \pi \nu\left[-D\left(\Delta_{6}^{1}+\Delta_{6}^{3}\right)-3 i \omega\right]
\end{aligned}
$$

where $\Delta_{n}^{m}$ is the sum of all distinct scalar products of pairs $q_{i}$ and $q_{i+m}$ of the $n$ incoming momenta which are separated by $m$ vertices,

$$
\Delta_{n}^{m}=\sum q_{i} \cdot q_{i+m}
$$

Note that in the derivation of Eq. (18) we have symmetrized over all incoming momentum variables.

If we use the above formulas to calculate the expansion of free energy $F(\omega)$ to three-loop order, the diagrams obtained are simply those we derived earlier using the trajectory language. The field theory enables us to associate an algebraic expression to each component in a diagram, and we obtain the results

$$
\begin{aligned}
F_{1} & =-\sum_{q_{1}} \ln \mathcal{P}_{1} \\
F_{2} & =\frac{1}{(2 \pi \nu)} \sum_{q_{1}, q_{2}} \frac{i \omega}{\mathcal{P}_{1} \mathcal{P}_{2}} \\
F_{3 a} & =\frac{2}{(2 \pi \nu)^{2}} \sum_{q_{1}, q_{2}, q_{3}} \frac{(i \omega)^{2}}{\mathcal{P}_{1}^{2} \mathcal{P}_{2} \mathcal{P}_{3}} \\
F_{3 b} & =-\frac{1}{(2 \pi \nu)^{2}} \sum_{q_{1}, q_{2}, q_{3}} \frac{\mathcal{P}_{1}}{\mathcal{P}_{1} \mathcal{P}_{2} \mathcal{P}_{3}} \\
F_{3 c} & =-\frac{1}{(2 \pi \nu)^{2}} \sum_{q_{1}, q_{2}, q_{3}} \frac{\mathcal{P}_{1}-2 i \omega}{\mathcal{P}_{1} \mathcal{P}_{2} \mathcal{P}_{3}} \\
F_{3 d} & =\frac{1}{4(2 \pi \nu)^{2}} \sum_{q_{1}, q_{2}, q_{3}} \frac{\left(\mathcal{P}_{12}-i \omega\right)^{2}}{\mathcal{P}_{1} \mathcal{P}_{2} \mathcal{P}_{3} \mathcal{P}_{123}} \\
F_{3 e} & =\frac{1}{2(2 \pi \nu)^{2}} \sum_{q_{1}, q_{2}, q_{3}} \frac{\left(\mathcal{P}_{12}-i \omega\right)\left(\mathcal{P}_{13}-i \omega\right)}{\mathcal{P}_{1} \mathcal{P}_{2} \mathcal{P}_{3} \mathcal{P}_{123}}
\end{aligned}
$$

where we use the notation

$$
\mathcal{P}_{1 \ldots n}=D\left(q_{1}+\ldots q_{n}\right)^{2}-i \omega .
$$

Of the above terms only $F_{1}, F_{3 b}$ and $F_{3 d}$ contribute in the unitary case. All diagrams contribute in the orthogonal case, and there is an extra factor of two arising from the two possible relative directions of the electron lines.

Symbolic expressions for $F$ similar to those in Eqs. (21) have been previously derived in Ref. 25 where the parameterization (13) has been used. Equations (21) derived within the parameterization (14) are valid for to both the ergodic and diffusive regimes.

Let us first check that these formulae reproduce the known results for the ergodic regime to this order. The ergodic regime corresponds to the zero-mode approximation in which we set all $q_{i}$ equal to zero. Doing this and differentiating twice with respect to $\omega$ yields

$$
\begin{aligned}
& R^{\text {ort }}(x)=\Re \mathrm{e}\left[\frac{1}{(i x)^{2}}+\frac{1}{(i x)^{3}}+\frac{3}{2(i x)^{4}}\right] \\
& R^{\mathrm{uni}}(x)=\Re \mathrm{e}\left[\frac{1}{2(i x)^{2}}\right]
\end{aligned}
$$

where $x=\pi \omega / A$ These are the correct expansions of the exact results 8 Indeed, for $K(t)$, which is the Fourier transform of $R(x)$, one has 8 in the region $0 \leq t \ll 1$ :

$$
\begin{aligned}
& K^{\text {ort }}(t)=t[2-\ln (t+1)] \approx 2 t-t^{2}+\frac{1}{2} t^{3} \\
& K^{\text {uni }}(t)=t \theta(1-t)=t
\end{aligned}
$$

Here $t$ is measured in units of $t_{H}=\hbar / \Delta$. (To compare the results of Eqs. (22b) and (23a), one uses the result that the inverse Fourier transform of $t^{n}$ is $i^{n+1} n ! / 2 x^{n+1}$.) Recall the famous result that there are no corrections to the universal RMT result in the unitary case to any order in perturbation theory. This happens due to the cancellation between rather than the absence of appropriate diagrammatic contributions, and is therefore a useful check on our calculation. Note also that the complete perturbative expansion for $R(\omega)$ would give, upon Fourier transformation, the exact $K(t)$ only for $t<1$. The perturbation theory cannot give the discontinuity point, $t=1$, because this is controlled by the non-trivial saddle point 2 .

We will now derive the leading-ordercontributions to $R(\omega)$ in 2 d. This was previously done 17 for the orthogonal case, and the form in the unitary case conjectured from a one-parameter scaling hypothesis. We replace the sums over $q$ in Eq. by integrals and use dimensional regularization to evaluate these integrals in $d=2+\epsilon$ dimensions. (Note that all the relevant integrals may be found in Ref. 24). Then we carefully take the limit $\epsilon \rightarrow 0$, keeping only the terms divergent in this limit. The two-loop orthogonal result is

$$
F_{2}^{\mathrm{ort}}(\omega)=\frac{\Delta^{2} L^{d}}{\pi^{2} \beta} \frac{1}{(2 \pi \nu)(2 \pi D)^{2}} \frac{(-i \omega)^{\epsilon+1}}{\epsilon^{2}}
$$

Taking the second derivative with respect to $\omega$, and noting that dimensionless conductance $g=G /\left(e^{2} / \pi h\right)=$ $4 \pi^{2} \nu D$, gives

$$
R_{2}^{\text {ort }}(\omega)=\frac{2 \Delta}{\pi g^{2}} \Re \mathrm{e}\left[\frac{(-i \omega)^{\epsilon-1}}{\epsilon}\right]
$$

Finally we let $\epsilon \rightarrow 0$ and note that $(-i \omega)^{\epsilon} / \epsilon$ becomes $\ln (-i \omega \tau)$. Hence

$$
R_{2}^{\mathrm{ort}}(\omega)=\frac{2 \Delta}{\pi g^{2}} \Re \mathrm{e}\left[\frac{\ln (-i \omega \tau)}{i \omega}\right]=-\frac{1}{g^{2} s}, \quad s \equiv \frac{\omega}{\Delta}
$$


The symplectic result would simply follow upon multiplying by $-1 / 2$.

Next we consider the three-loop unitary case. Careful evaluation of the integrals yields

$$
F_{3}^{\text {uni }}(\omega)=\frac{\Delta^{2} L^{d}}{\pi^{2} \beta} \frac{1}{(2 \pi \nu)^{2}(2 \pi D)^{3}} \frac{(-i \omega)^{3 \epsilon / 2+1}}{3 \epsilon^{2}} .
$$

Thus

$$
R_{3}^{\mathrm{uni}}(\omega)=\frac{\Delta}{\pi g^{3}} \Re \mathrm{e}\left[\frac{(-i \omega)^{3 \epsilon / 2-1}}{2 \epsilon}\right]
$$

which in the limit $\epsilon \rightarrow 0$ becomes

$$
R_{3}^{\mathrm{uni}}(\omega)=\frac{3 \Delta}{4 \pi g^{3}} \Re \mathrm{e}\left[\frac{\ln (-i \omega \tau)}{i \omega}\right]=-\frac{3}{8 g^{3} s} .
$$

Finally we calculate the three-loop orthogonal result:

$$
F_{3}^{\text {ort }}(\omega)=\frac{\Delta^{2} L^{d}}{\pi^{2} \beta} \frac{1}{(2 \pi \nu)^{2}(2 \pi D)^{3}} \frac{(4-3 \epsilon)(-i \omega)^{3 \epsilon / 2+1}}{3 \epsilon^{3}},
$$

so that

$$
R_{3}^{\mathrm{ort}}(\omega)=\frac{2 \Delta}{\pi g^{3}} \frac{(4+3 \epsilon)(-i \omega)^{3 \epsilon / 2-1}}{2 \epsilon^{2}},
$$

which in the limit $\epsilon \rightarrow 0$ gives

$$
R_{3}^{\mathrm{ort}}(\omega)=\frac{9 \Delta}{2 \pi g^{3}} \Re \mathrm{e}\left[\frac{\ln ^{2}(-i \omega \tau)+\ln (-i \omega \tau)}{i \omega}\right]=\frac{9}{4 g^{3} s}[1+2 \ln (s \Delta \tau)] .
$$

We now show that the same results can be obtained using a one-parameter scaling hypothesis in which the renormalized conductance, $g(\omega)$, is substituted into the lowest order $R(\omega)$ diagram. The latter has the form $R(\omega)=\left(\Delta^{2} / \pi^{2} \beta\right) \Re \mathrm{e} \sum_{q} \mathcal{P}_{1}^{-1}$, and substituting here $D=D_{0}+\delta D(\omega)$ gives upon the expansion in $\delta D$

$$
R(\omega)=\frac{\Delta^{2}}{\pi^{2} \beta}\left\{\sum_{q} \frac{1}{\left(D_{0} q^{2}-i \omega\right)^{2}}-2\left(\frac{\delta D}{D_{0}}\right) \sum_{q} \frac{D_{0} q^{2}}{\left(D_{0} q^{2}-i \omega\right)^{3}}+3\left(\frac{\delta D}{D_{0}}\right)^{2} \sum_{q} \frac{\left(D_{0} q^{2}\right)^{2}}{\left(D_{0} q^{2}-i \omega\right)^{4}}+\ldots\right\}
$$

We obtain the perturbative correction to the diffusion constant, $\delta D(\omega)$, from the $\beta(g)$ function which is defined by

$$
\beta(g)=\frac{d \ln g(L)}{d \ln L}
$$

To two-loop order the orthogonal and unitary beta functions are $\beta^{\text {ort }}(g)=2 / g$ and $\beta^{\text {uni }}(g)=2 / g^{2}$ from which it follows that

$$
\begin{gathered}
\frac{\delta D_{\text {ort }}}{D_{0}}=\frac{\delta g_{\text {ort }}}{g_{0}}=-\frac{2}{g_{0}} \ln (L / \ell) \rightarrow \frac{2}{g_{0}} \frac{(-i \omega)^{\epsilon / 2}}{\epsilon} \\
\frac{\delta D_{\text {uni }}}{D_{0}}=\frac{\delta g_{\text {uni }}}{g_{0}}=-\frac{2}{g_{0}^{2}} \ln (L / \ell) \rightarrow \frac{1}{g_{0}^{2}} \frac{(-i \omega)^{\epsilon}}{\epsilon}
\end{gathered}
$$

We can then substitute these results into Eq. (28), and use the following results for the $q$-integrals,

$$
\begin{aligned}
& \sum_{q} \frac{D_{0} q^{2}}{\left(D_{0} q^{2}-i \omega\right)^{3}}=-\frac{2+\epsilon}{8 \pi D}(-i \omega)^{\epsilon / 2-1} \\
& \sum_{q} \frac{\left(D_{0} q^{2}\right)^{2}}{\left(D_{0} q^{2}-i \omega\right)^{4}}=-\frac{4+3 \epsilon}{16 \pi D}(-i \omega)^{\epsilon / 2-1}
\end{aligned}
$$

We find that this exactly reproduces the results of Eqs. (24), (27) and (25), proving the validity of the scaling hypothesis to three-loop order. Note that the conjecture of Ref. 17 for $R_{3}^{\text {uni }}(\omega)$ does not produce the correct numerical factor in Eq. (26). The reason is that the calculation in Ref. 17 has been performed in exactly two dimensions where it was possible to take into account all weaklocalization logarithms but not logarithmic corrections arising from stronger mesoscopic divergences. To pick up all logarithmic corrections one must work in $d=2+\epsilon$ and take carefully the limit $\epsilon \rightarrow 0$, as we did here.

In conclusion, we have shown that the language of semiclassical trajectories suggests the most economical way of drawing diagrammatic corrections to spectral correlations in disordered electronic systems up to a high order of perturbation theory. We have introduced the weak diagonal approximation which includes the contributions of pairs of trajectories which are made from identical pieces joined together in different ways at some self-intersection (in real space) points. This gives physical meaning to the loop expansion of the field-theoretical 'free energy': the second derivative of this free energy with respect to frequency is the two-level correlation function. We have shown this directly by using the replicated nonlinear $\sigma$ model. Note that such a derivation does not work for the supersymmetric nonlinear $\sigma$ model as the free energy equals zero unless the supersymmetry is broken. Naturally, all the perturbative results may be reproduced within the supersymmetric model but in a much less economical way. Using the method described above, we have calculated the leading order contributions to the two-level correlation function in $2 d$ in the non-ergodic regime where the standard diagonal approximation gives vanishing results.

In the ergodic regime we have found that the loop expansion reproduces the deviation from linear in time behavior of the spectral form factor in the orthogonal case known from random matrix theory. In the unitary case, we have demonstrated that all such corrections are mutu- 
ally cancelled. The random matrix theory is believed to describe correctly level statistics of classically chaotic systems in the universal regime. ${ }^{5-7}$ It is therefore reasonable to conjecture that the weak diagonal approximation introduced here should be valid for generic chaotic systems. Although the universal regime is well understood within the supersymmetric approach, the weak diagonal approximation might be extended to the non-ergodic regime in chaotic systems.

\section{ACKNOWLEDGMENTS}

Work in Birmingham was supported by EPSRC grant GR/J35238. I.V.L. and B.L.A. thank the ITP in Santa Barbara for kind hospitality and partial support under NSF Grant No. PHY94-0719. R.A.S. and I.V.L. thank the Newton Institute at Cambridge for kind hospitality at the final stage of this work.

${ }^{1}$ N. Argaman, Y. Imry, and U. Smilansky, Phys. Rev. B 47, 4440 (1993).

${ }^{2}$ A. V. Andreev and B. L. Altshuler, Phys. Rev. Lett. 75, 902 (1995).

${ }^{3}$ O. Agam, A. V. Andreev, and B. L. Altshuler, Phys. Rev. Lett. 75, 4389 (1995).

${ }^{4}$ B. A. Muzykantskii and D. E. Khmelnitskii, JETP Letters 62, 76 (1995).

${ }^{5}$ A. V. Andreev, O. Agam, B. D. Simons, and B. L. Altshuler, Phys. Rev. Lett. 76, 3947 (1996).
${ }^{6}$ A. V. Andreev, B. D. Simons, O. Agam, and B. L. Altshuler, Nucl. Phys. B 482, 536 (1996).

${ }^{7}$ O. Bohigas, M. Giannoni, and C. Schmit, Phys. Rev. Lett. 52, 1 (1984).

${ }^{8}$ K. B. Efetov, Adv.Phys. 32, 53 (1983).

${ }^{9}$ F. Wegner, Z. Phys. B 35, 207 (1979).

${ }^{10}$ K. B. Efetov, A. I. Larkin, and D. E. Khmel'nitskii, Zh. Eksp. Teor. Fiz. 79, 1120 (1980) [Sov. Phys. JETP 52, 568 (1980)].

${ }^{11}$ M. C. Gutzwiller, J. Math. Phys. 12, 343 (1971).

12 M. V. Berry, Proc. R. Soc. Ser. A 400, 229 (1985).

${ }^{13}$ B. L. Altshuler and B. I. Shklovskii, Zh. Eksp. Teor. Fiz. 91, 220 (1986) [Sov. Phys. JETP 64, 127 (1986)].

14 A. I. Larkin and D. E. Khmelnitskii, Sov. Phys. Usp. 25, 185 (1982).

${ }^{15}$ D. E. Khmelnitskii, Physica B\&C 126, 235 (1984).

${ }^{16}$ S. Chakravarty and A. Schmid, Phys. Repts. 140, 193 (1986).

${ }^{17}$ V. E. Kravtsov and I. V. Lerner, Phys. Rev. Lett. 74, 2563 (1995).

${ }^{18}$ I. L. Aleiner and A. I. Larkin, Phys. Rev. B 54, 14423 (1996).

19 M. L. Mehta, Random matrices (Academic Press, Boston, 1991).

${ }^{20}$ O. Agam, I. V. Lerner, B. D. Simons, unpublished (1997).

${ }^{21}$ S. Hikami, Phys. Rev. B 24, 2671 (1981).

${ }^{22}$ A. Altland and Y. Gefen, Phys. Rev. B 51, 10671 (1995).

${ }^{23}$ B. L. Altshuler, V. E. Kravtsov, and I. V. Lerner, in Mesoscopic Phenomena in Solids, edited by B. L. Altshuler, P. A. Lee, and R. A. Webb, North-Holland, Amsterdam, 449 (1991).

${ }^{24}$ F. Wegner, Nucl. Phys. B 280, 193 (1987).

25 J. T. Chalker, I. V. Lerner, and R. A. Smith, J. Math. Phys. 37, 5061 (1996). 